\title{
In situ postmortem ethanol quantification in the cerebrospinal fluid by non-water-suppressed proton MRS
}

\author{
Zoelch, Niklaus ; Hock, Andreas ; Steuer, Andrea E ; Heimer, Jakob ; Kraemer, Thomas ; Thali, Michael J ;
} Gascho, Dominic

\begin{abstract}
Determination of the ethanol concentration in corpses with MRS would allow a reproducible forensic assessment by which evidence is collected in a noninvasive manner. However, although MRS has been successfully used to detect ethanol in vivo, it has not been applied to postmortem ethanol quantification in situ. The present study examined the feasibility of the noninvasive measurement of the ethanol concentration in human corpses with MRS. A total of 15 corpses with suspected alcohol consumption before demise underwent examination in a $3 \mathrm{~T}$ whole body scanner. To address the partial overlap of the ethanol and lactate signal in the postmortem spectrum, non-water-suppressed single voxel spectra were recorded in the cerebrospinal fluid (CSF) of the left lateral ventricle via the metabolite cycling technique. The ethanol signals were quantified using the internal water as reference standard, as well as based on a reference signal acquired in a phantom. The measured values were compared with biochemically determined concentrations in the blood (BAC) and CSF (CSFAC). In 8 of the 15 corpses a BAC above zero was determined (range $0.03-1.68 \mathrm{~g} / \mathrm{kg}$ ). In all of these 8 corpses, ethanol was measured in CSF with the proposed MRS protocol. The two applied MRS calibration strategies resulted in similar concentrations. However, the MRS measurements generally overestimated the ethanol concentration by $0.09 \mathrm{~g} / \mathrm{kg}(4 \%)$ to $0.72 \mathrm{~g} / \mathrm{kg}(45 \%)$ as compared with the CSFAC value. The presented MRS protocol allows the measurement of ethanol in the CSF in human corpses and provides an estimation of the ethanol concentration prior to autopsy. Observed deviations from biochemically determined concentrations are mainly explained by the approximate correction of the relaxation attenuation of the ethanol signal.
\end{abstract}

DOI: https://doi.org/10.1002/nbm.4081

Posted at the Zurich Open Repository and Archive, University of Zurich

ZORA URL: https://doi.org/10.5167/uzh-171279

Journal Article

Accepted Version

Originally published at:

Zoelch, Niklaus; Hock, Andreas; Steuer, Andrea E; Heimer, Jakob; Kraemer, Thomas; Thali, Michael J; Gascho, Dominic (2019). In situ postmortem ethanol quantification in the cerebrospinal fluid by non-water-suppressed proton MRS. NMR in Biomedicine, 32(5):e4081.

DOI: https://doi.org/10.1002/nbm.4081 


\section{In situ postmortem ethanol quantification in the cerebrospinal fluid by non-water-suppressed proton magnetic resonance spectroscopy}

\begin{tabular}{|r|l|}
\hline Journal: & NMR in Biomedicine \\
\hline Manuscript ID & NBM-18-0121.R2 \\
\hline Wiley - Manuscript type: & Research Article \\
\hline Date Submitted by the & n/a \\
\hline Complete List of Authors: & $\begin{array}{l}\text { Zoelch, Niklaus; Institute of Forensic Medicine, University of Zurich, } \\
\text { Department of Forensic Medicine and Imaging; Hospital of Psychatry, } \\
\text { University of Zurich, Department of Psychiatry, Psychotherapy and } \\
\text { Psychosomatics } \\
\text { Hock, Andreas; University and ETH Zurich, Institute for Biomedical } \\
\text { Engineering; Zurich University Hospital for Psychiatry, Department of } \\
\text { Psychiatry, Psychotherapy and Psychosomatics; Philips Healthcare } \\
\text { Deutschland } \\
\text { Steuer, Andrea; Institute of Forensic Medicine, University of Zurich } \\
\text { Heimer, Jakob; Institute of Forensic Medicine, University of Zurich, } \\
\text { Department of Forensic Medicine and Imaging } \\
\text { Kraemer, Thomas; University of Zurich, Institute of Forensic Medicine } \\
\text { Thali, Michael; University of Zurich, Institute of Forensic Medicine } \\
\text { Gascho, Dominic; Institute of Forensic Medicine, University of Zurich, } \\
\text { Department of Forensic Medicine and Imaging }\end{array}$ \\
\hline Keywords: \\
$\begin{array}{l}\text { MR Spectroscopy (MRS) and Spectroscopic Imaging (MRSI) Methods < } \\
\text { Methods and Engineering, Spectroscopic quantitation < MR Spectroscopy } \\
\text { (MRS) and Spectroscopic Imaging (MRSI) Methods < Methods and } \\
\text { Engineering, Quantitation < Post-acquisition Processing < Methods and } \\
\text { Engineering, Human study < Neurological < Applications }\end{array}$ \\
\hline
\end{tabular}

\section{SCHOLARONE" \\ Manuscripts}




\section{In situ postmortem ethanol quantification in the cerebrospinal fluid by non-water-suppressed proton magnetic resonance spectroscopy}

Niklaus Zoelch, Andreas Hock, Andrea E. Steuer, Jakob Heimer, Thomas Krämer, Michael J. Thali and Dominic Gascho

\section{Corresponding author}

Niklaus Zoelch

University of Zurich

Institute of Forensic Medicine

Winterthurerstrasse 190/52

CH-8057 Zürich

Tel +4144635 5611

Fax +41446356851

Email: niklaus.zoelch@uzh.ch

URL: http://www.irm.uzh.ch/

\section{Word count}

5755 Words

\section{Keywords}

- MR Spectroscopy (MRS)

- Quantification

- Postmortem

- Ethanol 
- Virtopsy

- Virtual autopsy

\section{Abbreviations}

Magnetic resonance spectroscopy (MRS)

Signal-to-noise ratio (SNR)

Postmortem interval (PMI)

Metabolite cycling (MC)

Echo time (TE)

Repetition time (TR),

Drive scale (DS)

Cerebrospinal fluid (CSF)

Blood-alcohol concentration (BAC)

Alcohol concentration in CSF (CSFAC)

Gray matter (GM)

White matter (WM)

Point resolved spectroscopy (PRESS)

Acetate (Ace)

Ascorbic-acid (Asc)

Aspartate (Asp)

Creatine (Cre)

Ethanol (EtOH)

Glutamate (Glu)

Glutamine (GIn)

Glycerophosphocholine (GPC)

Glycerol (Glyol)

Glycine (Gly)

Lactate (Lac) 


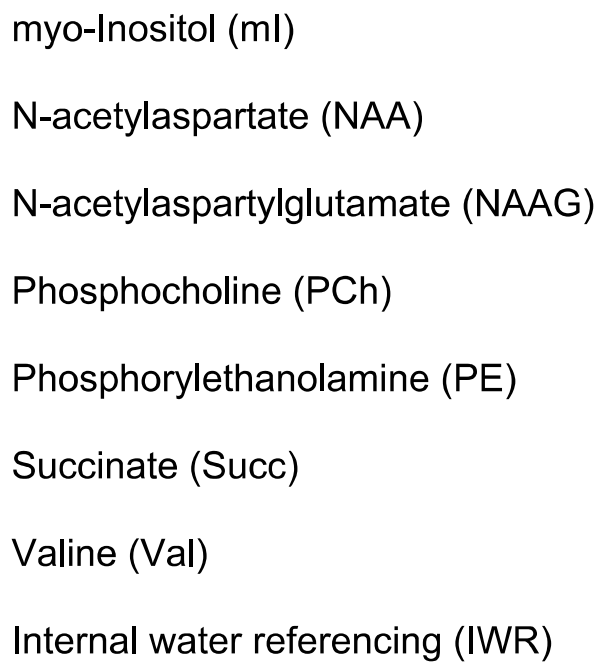




\begin{abstract}
The determination of the ethanol concentration in corpses with magnetic resonance spectroscopy (MRS) would allow a reproducible forensic assessment by which evidence is collected in a noninvasive manner. However, although MRS has been successfully used to detect ethanol in vivo, it has not been applied to postmortem ethanol quantification in situ. The present study examined the feasibility of the noninvasive measurement of the ethanol concentration in human corpses with MRS. A total of 15 corpses with suspected alcohol consumption before demise underwent examination in a $3 \mathrm{~T}$ whole body scanner. To address the partial overlap of the ethanol and lactate signal in the postmortem spectrum, non-water-suppressed single voxel spectra were recorded in the cerebrospinal fluid (CSF) of the left lateral ventricle via the metabolite cycling technique. The ethanol signals were quantified using the internal water as reference standard, as well as based on a reference signal acquired in a phantom. The measured values were compared to biochemically determined concentrations in the blood (BAC) and CSF (CSFAC). In 8 of the 15 corpses a BAC above zero was determined (Range: $0.03-1.68 \mathrm{~g} / \mathrm{kg}$ ). In all of these 8 corpses, ethanol was measured in CSF with the proposed MRS protocol. The two applied MRS calibration strategies resulted in similar concentrations. However, the MRS measurements generally overestimated the ethanol concentration by $0.09 \mathrm{~g} / \mathrm{kg} \mathrm{(4 \% )} \mathrm{to} 0.72 \mathrm{~g} / \mathrm{kg}(45 \%)$ as compared to the CSFAC value. The presented MRS protocol allows the measurement of ethanol in the CSF in human corpses and provides an estimation of the ethanol concentration prior to autopsy. Observed deviations form biochemically determined concentrations are mainly explained by the approximate correction of the relaxation attenuation of the ethanol signal.
\end{abstract}




\section{Introduction}

Ethanol is by far the most often encountered psychoactive substance in postmortem toxicology ${ }^{1}$ and, according to a study on the Swedish population ${ }^{2}$, plays an important role in approximately $28 \%$ of all unnatural deaths in Sweden. The quantitative determination of ethanol in a postmortem specimen is a routine procedure providing accurate and precise results. However, the measured concentrations are subject to factors such as ethanol absorption and distribution in the body at the time of death, postmortem diffusion and postmortem formation of ethanol. The femoral venous blood is considered the least susceptible to postmortem changes and is therefore recommended for toxicological analysis. Proton magnetic resonance spectroscopy (MRS) has been successfully used to detect ethanol in human brain tissue in vivo ${ }^{3-5}$. MRS has also been applied to examine alcohol tolerance ${ }^{4}$ and to demonstrate the metabolic alterations induced by chronic alcoholism ${ }^{6-10}$. Despite its potential, MRS has rarely been applied for postmortem measurements or forensic investigations ${ }^{11-13}$; however, noninvasive MRS offers several advantages for postmortem investigation. First, MRS allows a reproducible forensic assessment where evidence is collected in a noninvasive and nondestructive manner to complement or even supersede a conventional autopsy ${ }^{14}$. Furthermore, the extraction of samples from the human body after death is disallowed in some religious contexts. In these cases, noninvasive MRS provides medico-legal evidence without the violation of religious prohibitions or personal reservations. Finally, in cases of insufficient amounts of blood available for the analysis, as for example in neonates, in situ MRS can provide preliminary results without destroying precious evidence. However, there are some difficulties associated with measuring ethanol in the deceased using MRS. Due to anaerobic glycolysis, almost all postmortem spectra show a strong lactate signal, composed of a quadruplet at $4.1 \mathrm{ppm}$ and a doublet at $1.32 \mathrm{ppm}$. The latter is very close to the triplet signal of ethanol appearing at approximately $1.17 \mathrm{ppm}$, and hence the signal of ethanol, if present, is at least partly overlapped by the lactate signal. Therefore, 
a reliable assignment of the measured signals to the corresponding metabolites requires high spectral quality, i.e. a narrow linewidth and sufficient signal-to-noise ratio (SNR) ${ }^{15}$. Another important issue is data calibration, i.e., the conversion of the measured ethanol signals into an adequate measure of the amount of ethanol present in the investigated volume. Since MRS results might be compared to values measured by gas chromatography in postmortem analysis, concentrations expressed in standard units such as moles per kilogram must be calculated based on the measured MR signals ${ }^{16}$. Usually, this is achieved by using the signal from the tissue water as a reference. If the water and ethanol signals are acquired at an infinitely short echo-time (TE) and very long repetition time (TR), the moles of metabolite per mass of water can be derived without further assumptions. In practice, however, the achievable TE and TR are limited, and an estimate of the fully relaxed signals can be derived only by relaxation attenuation correction. In a forensic setting, the water relaxation times can vary considerably due to the different body temperatures ${ }^{17,18}$, postmortem intervals (PMI) and ages of the deceased ${ }^{19}$. Therefore, subject-specific measurements are necessary for this correction. Moreover, ethanol exists in at least two different molecular compartments in brain tissue with different mobilities ${ }^{3,20}$ : freely diffusing ethanol and a small pool of ethanol with restricted mobility due to its close association with macromolecules. The latter pool is characterized by very short $T_{2}$ relaxation times, therefore, very broad line widths, rendering it "invisible" to MRS using standard localization sequences with TE > $20 \mathrm{~ms}$. Different amounts of MRS-invisible ethanol in the brain tissue have been reported, ranging from 0 to $77 \% 21,22$. Additionally, the ethanol signal in the brain tissue might be contaminated by signals of lipids and macromolecules.

The aim of this study was to examine the feasibility of the noninvasive measurement of ethanol concentrations in human corpses by means of in situ MRS. We implemented nonwater-suppressed proton MRS via the metabolite cycling (MC) technique ${ }^{23-25}$ for postmortem measurements of ethanol in the cerebrospinal fluid (CSF) of the left lateral ventricle. In pure CSF, a small contamination from lipids and macromolecules and an MR visibility of ethanol 
of nearly $100 \%$ is expected. Compared to the blood-alcohol concentration (BAC), the ethanol concentration in the CSF is expected to be greater due to the difference in the relative water contents. Calibration of the MRS data was performed using the internal water as a reference with subject-specific measured water relaxation times and, for comparison, also based on a reference signal acquired in a phantom ${ }^{26}$. The latter does not depend on a correct estimation of the fully relaxed water signal. The ethanol concentrations estimated with MR were compared to the biochemically determined alcohol concentrations in the femoral venous blood and, if available, in the CSF obtained from the spinal canal.

\title{
Methods
}

\section{Subjects}

This study was performed with human cadavers. Ethical approval was waived by the responsible ethics committee of the Canton of Zurich (waiver number: 2015-0686). This article does not contain any studies with (living) human participants or animals performed by any of the authors. Scan data were acquired as part of a forensic judicial investigation.

\begin{abstract}
A total of 15 selected human corpses without injuries to the skull or brain and with suspected alcohol consumption before demise were measured at one point in time (Table 1). The rectal temperature of each corpse was measured before and after the MRS measurements. The mean corpse temperature is given in Table 2 and considered in the concentration measurements with MRS.
\end{abstract}

\section{MRS acquisition}

All experiments were performed using a $3 \mathrm{~T}$ whole-body MRI scanner (Achieva, Philips Healthcare, Best, The Netherlands). The body coil was used for excitation, whereas an 8channel SENSE head array coil (Philips Healthcare, Best, The Netherlands) was used for the 
reception of the signals. Anatomical $T_{1}$-weighted images (3D IR-TFE sequence, TR/TE/TI/FA: $9.4 \mathrm{~ms} / 4.6 \mathrm{~ms} / 600 \mathrm{~ms} / 8 \% 1 \mathrm{~mm}$ isotropic resolution) were acquired for spectroscopic voxel placement and tissue segmentation. The images were segmented into CSF, gray matter (GM), and white matter (WM) compartments using SPM8 ${ }^{27}$.

For the spectroscopic measurements, a voxel with a nominal voxel size of $40 \times 12 \times 10 \mathrm{~mm}^{3}$ was planned in the left lateral ventricle (Figure 1). The voxel position was adapted individually to maximize the CSF content. Prior to MRS data collection, magnetic filed shimming was performed (up to $2^{\text {nd }}$ order corrections) and the RF power was optimized on the localized voxel using a volume-selective power optimization ${ }^{28}$. This was done to assure an optimal flip angle and for data calibration using a reference signal acquired in a phantom. This method, in this investigation referred to as the drive scale (DS) method, is based on the principle of reciprocity and uses the voltage needed to obtain a certain flip angle in the measured voxel as a measure for the sensitivity and coil loading and therefore allows the correction of the differences in these quantities between the in situ and phantom measurements ${ }^{26}$.

In the first step, the water signal was measured (TE/TR $=35 / 7000 \mathrm{~ms}, 8$ averages); since the water signal obtained in the MC scans may not be valid for quantification. For data calibration with the drive scale method, one additional scan of the water signal with the body coil as the transceiver was performed with the same settings as above ${ }^{26}$. The water scans were followed by three non-water-suppressed, inner-volume saturated ${ }^{29,30}$, single-voxel, pointresolved spectroscopy (PRESS) ${ }^{31}$ proton MRS scans using the MC technique ${ }^{23,25}$ (TE/TR 35/3000 ms, readout duration $1024 \mathrm{~ms}$, bandwidth $2000 \mathrm{~Hz})$. In total, $384(3 \times 128)$ metabolite spectra were collected for each subject. Inner-volume saturation was applied to reduce the problems arising from the fact that the methylene protons $\left(\mathrm{CH}_{2}\right)$ of ethanol resonating at 3.67 ppm and the methyl protons at $1.17 \mathrm{ppm}\left(\mathrm{CH}_{3}\right)$ are not excited equally over the whole voxel due to chemical shift displacement ${ }^{32}$. Inner-volume saturation was applied for all spectroscopy measurements and resulted in an effective voxel size of $27.3 \times 9.8 \times 6.8 \mathrm{~mm}^{3}$. 
The water relaxation properties were finally measured with an echo time series $(T E=35,94$, $162,240,332,445,592,800 \mathrm{~ms})$ with $\mathrm{TR}=7 \mathrm{~s}$, and a series of inversion recovery measurements $(T E=35 \mathrm{~ms}, \mathrm{TR}=7 \mathrm{~s}$ ) with inversion times of $35,412,827,1291,2421$, 3133 , and $4000 \mathrm{~ms}$.

\section{MRS analysis}

Proton MRS using the MC technique allows for the simultaneous analyses of water and metabolite spectra by employing an adiabatic inversion pulse prior to the localization sequence that alternately inverts the up-field or down-field metabolites. The high SNR water peak in each individual spectrum enables frequency alignment and phase correction on each spectrum. The sum of all spectra yield the water peak, while the difference between the upand down-field inteverted spectra yields the metabolite spectrum 23-25,33. Postprocessing included downsampling to 1024 points and singular-value decomposition-based coil combination ${ }^{34}$ implemented in MRecon (Gyrotools, Zurich, $\mathrm{CH}$ ). The averaged signals were corrected for eddy current induced line-shape distortions using the water signal phase ${ }^{35}$. Additionally, all averaged metabolite signals were truncated and zero-filled after $200 \mathrm{~ms}$. A Hankel singular value decomposition water filter was applied to remove the remaining water peak and achieve a flat baseline. Each spectrum was manually shifted at the end so that the center of the lactate duplet appeared at $1.32 \mathrm{ppm}$.

Metabolite spectra were fitted using LCModel (Version 6.3) ${ }^{36}$ between 0.2 and 4.0 ppm using a simulated basis set. The basis set included acetate (Ace), alanine, ascorbic-acid (Asc), aspartate (Asp), creatine (Cre), ethanol (EtOH), y-aminobutyric acid, glucose, glutamate (Glu), glutamine (GIn), glutathione, glycerophosphocholine (GPC), glycerol (Glyol), glycine (Gly), lactate (Lac), myo-inositol (ml), N-acetylaspartate (NAA), N-acetylaspartylglutamate (NAAG), phosphocholine (PCh), phospho-creatine, phosphorylethanolamine (PE), scylloinositol, succinate (Succ), taurine and valine (Val), as suggested by lth et al. ${ }^{11}$ for measurements up to three days postmortem. For subject No. 8, with an estimated PMI of 
more than three days, the same basis set was used, since the fit residuals showed no signs of the additional metabolites identified by Ith et al. ${ }^{11}$ in spectra obtained 15 days postmortem. Basis set simulations were performed using FID-A (Version 1.1), an open-source software toolkit for the simulation and processing of MRS data ${ }^{37}$. 2D simulations were carried out over the whole voxel volume with a spatial resolution of $40 \times 40$ points in the directions of the refocusing pulses and using the actual pulse shape of the refocusing pulses. To obtain the basis set for LCModel, the spectra within the effective voxel size were summed, assuming ideal saturation bands. Ethanol was simulated with a J-coupling constant of $7.1 \mathrm{~Hz}{ }^{38}$.

Within LCModel, the Lac and Cre signals were used for the preliminary analysis, as the usual landmarks are missing or weak. The search for first- and zero-order phase correction was limited (SDDEGZ=4 and SDDEGP =4), and the starting value for the frequency shift was set to zero, since the spectra were already eddy current corrected and shifted in the postprocessing. The water concentration used within LCModel was set to the pure water concentration $(\mathrm{WCONC}=55556)$ and the relaxation attenuation correction was omitted to allow subject-specific corrections later on.

The echo time series measured to estimate the $T_{2}$ relaxation times of the water resonance for each subject were first fitted with a mono exponential decay. If the fit was not adequate $(R<0.996)$, a biexponential decay was fitted. The shorter relaxation times were then attributed to water in the parenchyma ( $\mathrm{GM}$ and WM tissue), $T_{2, \mathrm{PA}}$, whereas the longer time was attributed to water in the CSF, $T_{2, C S F}$. Inversion time series were fitted with a mono exponential decay, resulting in $T_{1 \text {, mix. }}$ Multiple regression analysis was used to investigate the temperature and CSF fraction dependence of the measured relaxation times over all subjects.

\section{Concentrations determined by MRS}

The ethanol concentrations were estimated based on the fitted peak areas $S_{\text {EtOH }}$ using the measured internal water signal $\mathrm{S}_{\mathrm{H} 2 \mathrm{O}}$ as a reference and the drive scale method. In both 
cases, the ethanol concentrations are derived in grams of ethanol per $\mathrm{kg}$ of the solvent, which is the (MR visible) water in the measured volume in this case.

For the calculation of the ethanol concentrations with internal water referencing (IWR) Equation 1 was applied. Equation 1 is derived from Gasparovic et al. ${ }^{39}$, where an equation for the determination of brain metabolite concentrations in heterogeneously composed voxels is proposed (Eq. 8 therein). This equation is intended for metabolites, which are only measurable in the parenchyma, and therefore the corresponding metabolite signals are referenced only to the water signal from the parenchyma to derive concentrations relative to the mass of brain water excluding CSF. For the investigation presented here, ethanol is present in both brain tissue and CSF and, therefore, the factor $\left(1-f_{\mathrm{csf}}\right)$ in Gasparovic's expression needs to be omitted in order to reference the ethanol signal to all water in the measured voxel. $\mathrm{M}_{\mathrm{H} 20}$ is the molar mass of water $(0.018015 \mathrm{~kg} / \mathrm{mol})$ and $\mathrm{M}_{\mathrm{EtOH}}$ the molar mass of ethanol given in $\mathrm{g} / \mathrm{mol}(46.07 \mathrm{~g} / \mathrm{mol})$ to directly obtain $\mathrm{g} / \mathrm{kg}$ concentrations. The number of MR-visible protons per molecule of the corresponding metabolite, 2 for water and 5 for ethanol, is denoted by $l$.

$$
\mathrm{c}_{\mathrm{EtOH}}^{\mathrm{IWR}}=\frac{S_{E t O H}}{S_{\mathrm{H}_{2} \mathrm{O}}} \cdot \frac{R_{\mathrm{H}_{2} \mathrm{O}}}{R_{E t O H}} \cdot \frac{l_{\mathrm{H}_{2} \mathrm{O}}}{l_{\mathrm{EtOH}}} \cdot \frac{M_{E t O H}}{M_{\mathrm{H}_{2} \mathrm{O}}}
$$

The relaxation attenuation factor $R_{\mathrm{H} 2 \mathrm{O}}$ for the water signal is given in Equation 2, where $f_{\text {csf_mol }}$ is the molar fraction of water attributable to the CSF ${ }^{39}$. The measured relaxation times given in Table 2 were used to calculate $\mathrm{R}_{\mathrm{H} 2 \mathrm{O}}$ for each corpse individually.

$$
R_{H 2 O}=\left(1-\exp \left(-\frac{T R}{T_{1, m i x}}\right)\right)\left(\left(1-f_{c s f_{-} m o l}\right) \exp \left(-\frac{T E}{T_{2, P A}}\right)+f_{c s f_{-} m o l} \exp \left(-\frac{T E}{T_{2, C S F}}\right)\right)
$$

The relaxation attenuation correction of the ethanol signal was performed using reported tissue-specific ethanol methyl $T_{1}$ and $T_{2}$ values determined at $3 T$. The longitudinal relaxation time constants of $\mathrm{T}_{1, \mathrm{EtOH} \_\mathrm{GM}}=\mathrm{T}_{1, \mathrm{EtOH} \_\mathrm{WM}}=2000 \mathrm{~ms}$, and $\mathrm{T}_{1, \mathrm{EtOH} \_\mathrm{CSF}}=4400 \mathrm{~ms}$ were taken from 40. $\mathrm{T}_{2, \mathrm{ETOH}}$ CSF was estimated with $725 \mathrm{~ms}$ as measured by Flory et al. ${ }^{41}$ in dilute solution. From the same work, $T_{2, E t O H} \mathrm{WM}_{\mathrm{M}}=131 \mathrm{~ms}$ and $\mathrm{T}_{2, \mathrm{EtOH} \_\mathrm{GM}}=237 \mathrm{~ms}$, estimated in the primate 
brain, were adopted herein. To be able to perform tissue-specific relaxation correction for ethanol, equal relative densities were assumed in the three different tissue types. This rough approximation allows using volume fractions instead of mole fractions in Equation 3:

$$
\begin{gathered}
R_{E t O H_{-} j}=\exp \left(-\frac{T E}{T_{2, E t O H_{-}}}\right)\left(1-\exp \left(-\frac{T R}{T_{1, E t O H_{-} j}}\right)\right) \\
R_{E t O H}=f_{C S F} R_{E t O H_{-} C S F}+f_{G M} R_{E t O H_{-} G M}+f_{W M} R_{E t O H_{-} W M}
\end{gathered}
$$

Ethanol concentrations estimated based on a reference signal $S_{\text {ref,cal }}$ acquired in a phantom were calculated using the drive scale method as described in detail in ${ }^{26}$. The scaling factor DS is proportional to the output voltage of the RF transmitter needed to obtain a certain flip angle and was determined with a volume-selective power optimization ${ }^{28}$ with the body coil as the transceiver. To properly reference $S_{\mathrm{EtOH}}$ detected with the eight-channel phased array coil to the reference signal, a conversion factor calculated using the water signal detected once with the body coil as the transceiver $\left(\mathrm{S}_{\mathrm{H} 2 \mathrm{O}, \text { body }}\right)$ and once using the body coil for transmission and the phased array coil for detection $\left(\mathrm{S}_{\mathrm{H} 20}\right)$ is necessary ${ }^{42,43}$. Calibration measurements in the phantom are performed with the body coil as the transceiver. The applied equation (Equation 4 ) is adapted from ${ }^{26}$

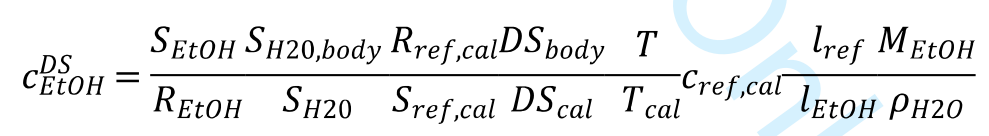

The subscript cal in Equation 4 denotes quantities obtained in the phantom measurement, and the subscript ref quantities dependent on the measured reference compound present in the phantom. In this study, water is the reference compound measured in the phantom at $T_{\text {cal }}$ $=295 \mathrm{~K}$ with a concentration $\mathrm{c}_{\text {ref,cal }}$ of $55.4 \mathrm{~mol} / \mathrm{l}$ (pure water). Temperature differences between the corpses and the phantom were corrected using the average temperature as given in Table 2.

The concentration unit that is most straightforwardly determined with the drive scale method is moles per measured volume (mol/l). For the comparison with the BAC given in $\mathrm{g}$ per $\mathrm{kg}$ of solvent, a division with the assumed water density in the voxel $\left(\rho_{H 2 O}\right)$ is necessary. Here $\rho_{H 2 O}$ 
was estimated with Equation 5 , where $\mathrm{d}_{\mathrm{H} 2 \mathrm{O}}$ is the density of pure water at room temperature, $f_{m}$ the volume fractions resulting from tissue segmentation and $\alpha$ the assumed relative water densities taken from ${ }^{44}$.

$$
\rho_{H 2 O}=d_{H 2 O}\left(f_{G M} \alpha_{G M}+f_{W M} \alpha_{W M}+f_{C S F} \alpha_{C S F}\right)
$$

Since voxels were placed in the left lateral ventricle, the measured positions are near the middle of the coil, where the deviations of the transmit field from the reception field are expected to be small. Therefore, the according corrections for the drive scale method mentioned in ${ }^{26}$ were omitted.

Additionally, the water concentration in the measured voxel was determined with the drive scale method. For that, Equation 6, providing the molar water concentration, was applied:

$$
c_{H 2 O}^{D S}=\frac{S_{H 20, b o d y} R_{\text {ref,cal }} D S_{\text {body }} T}{R_{H 20} S_{\text {ref,cal }} D S_{\text {cal }} T_{c a l}} c_{r e f, c a l} \frac{l_{\text {ref }}}{l_{H 2 O}}
$$

The water concentration $c_{H 2 O}^{D S}$ was compared to the water concentration estimated using the information from the tissue segmentation $c_{H 2 O}^{s e g}$ (Equation 7), with 55.40 the concentration of pure water at room temperature in $\mathrm{mol} / \mathrm{l}$.

$$
c_{H 2 O}^{s e g}=\frac{\rho_{H 2 O}}{M_{\mathrm{H}_{2} \mathrm{O}}}=55.40\left(f_{G M} \alpha_{G M}+f_{W M} \alpha_{W M}+f_{C S F} \alpha_{C S F}\right)
$$

If $c_{H 2 O}^{s e g}$ (Eq. 7) coincides with the water concentration determined with the drive scale method $\left(c_{H 20}^{D S}\right.$, Eq. 6), $c_{E t O H}^{I W R}$ and $c_{E t O H}^{D S}$ will yield the exact same result. Comparing $c_{H 20}^{D S}$ and $c_{H 2 O}^{s e g}$ therefore allows a general comparison of the two quantification approaches, also in cases where no ethanol was measured.

\section{Biochemical Analysis}

Blood samples from a femoral vein were collected immediately prior to autopsy from all subjects. In ten corpses, cerebrospinal fluid was additionally obtained during autopsy from 
the spinal canal. Autopsy was performed 2 to 3 hours after the MR measurement on average. All samples were stored at $20{ }^{\circ} \mathrm{C}$ until analysis. Ethanol quantification was performed by headspace gas chromatography coupled to flame ionization detection (Perkin Elmer, Glarus $580 \mathrm{GC}$, Lausanne, Switzerland). Fourfold determination was performed for each sample using duplicates on two different chromatographic systems (method 1: GC column J\&W DB-ALC2; 30 m; 0.320 mm ID; $1.20 \mu \mathrm{m}$, internal standard propionitrile; method 2 GC column Restek Rtx®-Wax; 30 m; 0.320 mm ID; $1.20 \mu \mathrm{m}$, internal standard acetonitrile). For each measurement $0.25 \mathrm{~g}$ of whole blood or CSF was weighed into headspace autosampler vials followed by the addition of $0.75 \mathrm{~g}$ of the internal standard solution (concentration $0.01 \%, v / v$ ). Quantitative results were obtained by the calculation of the ethanol peak area over the internal standard area against an external five-point calibration curve ranging from 0.1 to $3 \mathrm{~g} / \mathrm{kg}$. The accuracy was reviewed in each batch through the monitoring of three concentration levels of commercially available quality control samples $(0.5 \mathrm{~g} / \mathrm{kg}, 1 \mathrm{~g} / \mathrm{kg}, 2 \mathrm{~g} / \mathrm{kg}$, criteria bias $<5 \%$ of target concentration) and the internal deviations of fourfold measurements (criteria relative standard deviation $<5 \%$ ).

For comparison, an estimate of the ethanol concentration in the CSF based on the BAC (eCSFAC) was calculated using a regression equation (Equation 8) based on data from 509 human corpses ${ }^{45}$.

$$
e C S F A C=-0.11+1.35 \cdot B A C
$$

\section{Results}

All measured ethanol concentrations are shown in Figure 2 and summarized in Table 2 together with the estimated water relaxation times. Due to the different sizes and shapes of the ventricle, the measured MRS voxels contained different amounts of CSF. 
In 8 of the 15 corpses, a BAC above zero was measured with gas chromatography. In all of these 8 corpses, it was possible to detect ethanol in the CSF with the proposed MRS protocol.

If ethanol was detected in the CSF samples taken during autopsy (CSFAC), the measured concentration was in all cases higher than the BAC and agreed mostly with the eCSFAC values. Pronounced deviations $(>10 \%)$ of the expected value were measured in corpses No. 13 and 3 (Table 2).

The agreement between the biochemically determined concentrations and the MRS measurement is assessed in Figure 3 with Bland-Altman plots and scatterplots.

The mean difference between $c_{E t O H}^{I W R}$ and CSFAC is not significantly different from 0 and a strong positive correlation ( $r=0.91, p<0.014$, by Pearson) was observed (Figure 3.A.). The $c_{E t O H}^{I W R}$ concentration differed by up to $0.72 \mathrm{~g} / \mathrm{kg}$ from the measured CSFAC (Corpse No. 15), which is $45 \%$ percent of the measured CSFAC value. The smallest deviation is observed in corpse No. $6,0.09 \mathrm{~g} / \mathrm{kg}(4 \%)$. The difference between $c_{E t O H}^{I W R}$ and CSFAC tends to be more pronounced for lower corpse temperatures. However, this correlation is not significant ( $r$ $=0.64, \mathrm{p}=0.17)$. Additionally, for the difference between $c_{E t O H}^{D S}$ and CSFAC a nonsignificant correlation with temperature was observed $(r=0.75, p=0.09)$.

The scatterplots in Figure $3 \mathrm{~B}$ reveal a strong positive correlation of $c_{E t O H}^{I W R}$ with BAC $(r=0.94$, $\mathrm{p}<0.001$ ), and a significant mean difference is observed in the Bland-Altman plot. $c_{E t O H}^{I W R}$ is higher than the corresponding $\mathrm{BAC}$ in all cases. The median $c_{E t O H}^{I W R}$ to $\mathrm{BAC}$ ratio is 1.38 and the median CSFAC to BAC ratio is 1.22 . Using MRS, similar ethanol concentrations are determined with internal water as the reference $\left(c_{E t O H}^{I W R}\right)$ and with the drive scale method $\left(c_{E t O H}^{D S}\right.$ ) (Figure $3 \mathrm{C}$, correlation $(r=0.995, p<0.0001))$.

The maximum deviation of $c_{E t O H}^{D S}$ from $c_{E t O H}^{I W R}$ as a percentage of the mean of the two values is $9 \%(0.09 \mathrm{~g} / \mathrm{kg})$, observed in corpse No. 11 . The deviations between the drive scale method and IWR are best shown in Figure 4, where differences in water concentrations estimated using the drive scale method $c_{H 2 O}^{D S}$ and based on segmentation $c_{H 2 O}^{\text {seg }}$ in all subjects are plotted. The observed correlation with the CSF fraction is not significant $(r=0.2, p=0.09)$. However, 
excluding outlier No. 11, which lies 1.5 times the interquartile range below the lower quartile, reveals a significant correlation $(0.54, p<0.003)$.

In Figure 5, a representative spectrum measured in corpse No. 13 and its decomposition into fitted metabolite signals is shown. The ethanol signal is clearly visible, with the quadruplet at approximately $3.64 \mathrm{ppm}$ and the triplet at $1.17 \mathrm{ppm}$ partly overlaid by the lactate peak.

Multiple regression analysis revealed the dependence of the measured $T_{1}$ and $T_{2}$ csf on the temperature and CSF fraction over all subjects. In the case of $T_{1}$, the two predictors explained $82.5 \%$ of the variance $\left(R^{2}=0.825, F(2,12)=28.22, p<0.0001\right)$. Both the temperature $(\beta=16, p<0.01)$ and CSF fraction $(\beta=981, p<0.0001)$ were significant predictors for $T_{1}$. In the case of $T_{2}$ csf, $61.3 \%$ of the variance $\left(R^{2}=0.613, F(2,12)=9.5, p<0.01\right)$ was explained by the temperature $(\beta=23, p<0.05)$ and CSF fraction $(\beta=955, p<0.01)$.

\section{Discussion}

The present study explored the feasibility of estimating ethanol concentrations noninvasively in human corpses with MRS. To the best of our knowledge; this is the first attempt of this type in a postmortem setting. The applied MR protocol using the MC technique provides postmortem spectra from small voxels, which allowed resolving the ethanol signal from overlapping lactate signals in a brain region containing mostly CSF even in cases with low ethanol levels.. However, the measured concentrations differ from the biochemically measured values in the CSF. The observed deviations tend to be more pronounced for lower temperatures.

The advantages of non-water-suppressed MC PRESS compared to the more common water-suppressed PRESS measurements might be fewer in post-mortem applications than in vivo due to the absence of field changes caused by subject motion, respiration, and blood flow. The observed resolution of lactate from ethanol is partly due to the fact that the observed linewidths in the postmortem CSF measurements in the absence of flow are generally small. Nonetheless, the MC technique allows an optimal correction of the 
frequency drifts resulting from temporal scanner instability, which can be severe during long scan times. The measurement with $\mathrm{MC}$ at $\mathrm{TE}=30 \mathrm{~ms}$ has distinct advantages compared to protocols exploiting the difference in the J-coupling evolution to separate the ethanol from lactate by measuring longer TE, for example at TE $=144 \mathrm{~ms}$, to invert the lactate resonance at $1.32 \mathrm{ppm}$. The potentially increased separability of such approaches is compromised by the signal loss due to $\mathrm{T}_{2}$ relaxation as well as $\mathrm{J}$-coupling evolution. Additionally, quantification at longer TE depends even more on an accurate estimation of $T_{2}$ for ethanol for the relaxation attenuation correction than at short TE.

The ethanol concentration measured in different tissue types depends on the relative water content in the tissue ${ }^{46}$, physiological processes occurring before death, i.e. absorption and elimination processes, and the progression of postmortem changes. The average ratio of 1.22 for the CSFAC and BAC determined in the present study is close to the ratio of the water contents of the CSF $(\approx 0.99)$ and blood $(0.8)^{47}$, which is $\approx 1.24$.

In principle, similar results are expected for CSFAC and $c_{E t O H}^{I W R}$ as described in this study. However, there are subtle differences between the two quantities that lead to different concentrations even under the assumption of no measurement errors. For $c_{E t O H}^{I W R}$ measured in a pure CSF voxel and CSFAC, the deviations are expected to be small, but since the weight of the sample is considered in the biochemical analysis, deviations of the CSF density from the pure water density would bias the comparison. In a mixed-tissue voxel, $c_{E t O H}^{I W R}$ might still be a valid estimation for CSFAC, if the MR-visibilities of both ethanol and water are not different in the GM and WM tissue from that of CSF. A small fraction of the water is known to be MRinvisible in WM tissue ${ }^{48}$, which may have lead in this case to an overestimation of $c_{E t O H}^{I W R}$ compared to CSFAC. However, some fraction of the ethanol is also expected to be MRinvisible ${ }^{3}$ due to interactions with membranes, and therefore the $c_{E t O H}^{I W R}$ could be an underestimation of CSFAC. The deviations observed in our study appear to be too pronounced to be explained solely by the fraction water or ethanol binding differences between CSFAC and $c_{E t O H}^{I W R}$ mentioned above. Regardless of the underlying factors, $c_{E t O H}^{I W R}$ in 
this study generally overestimates the CSFAC concentration, which is demonstrated by the high mean $c_{E t O H}^{I W R}$ to $\mathrm{BAC}$ ratio.

There are several potential reasons for the overestimation of the ethanol concentrations in this work. For example, the $T_{1}$ times for water protons in the CSF measured herein are lower than the in vivo values reported in the range of $3-4 s{ }^{49,50}$. This may partially be explained by postmortem effects and lower corpse temperatures. However, the return to the baseline of the water signal in the inversion recovery experiments was insufficiently sampled in this study, since the maximum inversion time was comparable to the estimated maximum observable $T_{1}$ in the CSF ${ }^{51}$. This limits the precision and accuracy of the measured $T_{1}$ times. Nevertheless, inaccuracies in the $\mathrm{T}_{1}$ (and $\mathrm{T}_{2}$ ) of the water signal influence only the $c_{E t O H}^{I W R}$ values, and not ethanol concentrations derived with the drive scale method, and therefore may not explain the observed difference between the CSFAC and the MRS values.

However, the $c_{E t O H}^{I W R}$ and $c_{E t O H}^{D S}$ values both highly depend on the $\mathrm{T}_{1}$ and $\mathrm{T}_{2}$ values assumed for the relaxation attenuation correction of the ethanol signal. Based on the observed temperature dependence of the water relaxation times, a change of the actual ethanol relaxation time by almost a factor of two seems reasonable over the measured temperature range. With the chosen settings for TE and TR and over the expected range of ethanol relaxation times, deviations of the assumed $T_{1, E t O H}$ from the actual $T_{1}$ of ethanol would have a pronounced influence on the determined concentration. We therefore think that the main reason for the observed deviations, as well as a main limitation of this study, is that the $T_{1}$ relaxation attenuation correction of the ethanol signal was based on literature values measured at body temperature as opposed to measured ethanol $T_{1}$ values. This reasoning is supported by the apparent temperature dependence of the difference between the MRSmeasured concentrations and CSFAC. Lower $\mathrm{T}_{1, \mathrm{EtOH}}$ values used for correction at lower temperatures would result in reduced MRS-measured concentrations and partially explain the observed deviations of the CSFAC. To date, few relaxation time measurements of ethanol in humans have been published ${ }^{3,21,40,52}$, and the $T_{1}$ times of ethanol might also change with the age of the deceased ${ }^{19}$ and the PMI. Therefore, an estimation of the actual 
$\mathrm{T}_{1, \mathrm{ETOH}}$ in each subject based on literature values alone is difficult. While technically demanding and requiring more scan time, measuring the actual $\mathrm{T}_{1, \mathrm{ETOH}}$ in each subject would be expected to improve the agreement between the MRS results and CSFAC. Alternatively, a protocol with a longer TR and a very short TE, for example with STEAM localization, could be used to minimize the effects of signal relaxation. Further studies might also consider using even smaller voxel sizes to reduce GM and WM contamination. However, due to the rectangular voxel shape and brain swelling, which is often observed postmortem, it would be unlikely to avoid the GM and WM in all cases.

The basis set spectra used with LCModel to quantify metabolites in this study were simulated taking into account spatial localization and actual RF pulse shapes. This appeared to improve the accuracy of the spectral fitting, since fitting with basis sets simulated with ideal pulses and disregarding voxel localization resulted in more pronounced residuals and extra side peaks in the lineshape function in LCModel. Nonetheless, inaccuracies in the macromolecular signal and baseline estimation in voxels with a smaller CSF fraction remain, in addition to the general uncertainty in the fitting of low- concentration metabolites.

Since inaccuracy in the relaxation attenuation correction and remaining inaccuracies in the fit of the ethanol signal affect $c_{E t O H}^{I W R}$ and $c_{E t O H}^{D S}$ in the same way, similar values are obtained using both quantification approaches. Differences between the two calibration strategies are expected to be mainly due to inaccuracies in the relaxation attenuation correction of the water signal and an incorrectly estimated $\rho_{H 2 O} . c_{E t O H}^{D S}$ depends on the validity of the used relative water densities and the correctness of the tissue segmentation, both of which might be affected by postmortem changes, such as decomposition and a low corpse temperature. In this respect, the observed tendency of the difference between $c_{H 2 O}^{\text {seg }}$ and $c_{H 2 O}^{D S}$ to increase with decreasing CSF fraction can be explained in two ways. Either the actual water densities are overestimated for voxels with low CSF fractions, or the relaxation attenuation correction for the water signal is inadequate in this case, e.g., caused by an underestimation of $T_{1, \text { mix }}$ or an overestimation of $T_{2, p a}$. Since a general postmortem decrease of the relative water density in the brain tissue seems unlikely, we suspect the latter to cause the observed dependency. 
Since it does not depend on the water signal, the drive scale method is not subject to this potential pitfall. However, the differences between the two calibration approaches are small compared to the deviations from the CSFAC and more measurements may be needed to elucidate whether the assumed relative water densities measured in vivo are adequate for postmortem measurements in mixed-tissue voxels. The possibility of measuring the water concentration in the investigated voxel with the drive scale method could be of additional value for postmortem applications, where changes in water concentrations are expected in edema or due to tissue decomposition and replacement by water.

This study demonstrates that the MRS derived ethanol concentrations in CSF strongly correlate with biochemically derived BAC values. A previous MRS study on living subjects reported $r=0.58$ for the correlation of the ethanol/NAA ratios measured in brain tissue with BAC values. However, the authors measured a smaller range of BAC values ${ }^{3}$ (estimated BAC range in whole blood: $0.37-0.81 \mathrm{~g} / \mathrm{kg}$ ). After the intake of ethanol, the ethanol concentration reaches its maximum in the CSF only after the maximum has been reached in venous blood ${ }^{1}$ which must be taken into account, when measured concentrations are interpreted in forensic investigations. The ethanol concentrations in the CSF obtained in a suboccipital region were reported to be relatively stable against postmortem changes, comparable to the concentrations measured in venous blood. No postmortem formation of ethanol and no diffusion of ethanol directly from the stomach was observed ${ }^{53}$, which makes the CSF a valuable specimen for postmortem investigations.

Currently, access to MRI scanners is limited in most forensic institutions, and internal or external toxicological analyses provide results with precision and accuracy. However, with MRS the collection of specimens that are exhausted in the analysis is avoided, while results are available before the onset of a potential autopsy. Moreover, in vivo MRS has been shown to detect differences in scyllo-inositol concentrations between non-detoxified chronic alcoholics compared to healthy controls ${ }^{6}$ and was able to show increased MR visibility of ethanol in alcohol-tolerant drinkers ${ }^{4}$. If such findings could be reproduced postmortem with a protocol that yields sufficient sensitivity for single-corpse analyses, MRS could potentially 


\section{Acknowledgments}

We thank all the morgue technicians at our institute for the specimen collection and Dr. Andrea Oestreich for his insight into blood ethanol quantification.

\section{References}

1. Kugelberg FC, Jones AW. Interpreting results of ethanol analysis in postmortem specimens: a review of the literature. Forensic Science International. 2007;165(1):10-29.

2. Sjögren $\mathrm{H}$, Eriksson A, Ahlm K. Role of alcohol in unnatural deaths: a study of all deaths in Sweden. Alcoholism: Clinical and Experimental Research. 2000;24(7):1050-1056.

3. Fein G, Meyerhoff DJ. Ethanol in human brain by magnetic resonance spectroscopy: correlation with blood and breath levels, relaxation, and magnetization transfer. Alcoholism: Clinical and Experimental Research. 2000;24(8):1227-1235.

4. Chiu T-M, Mendelson JH, Woods BT, Teoh SK, Levisohn L, Mello NK. In vivo proton magnetic resonance spectroscopy detection of human alcohol tolerance. Magnetic Resonance in Medicine. 1994;32(4):511-516.

5. Hanstock C, Rothman D, Shulman R, Novotny Jr E, Petroff O, Prichard J. Measurement of ethanol in the human brain using NMR spectroscopy. Journal of Studies on Alcohol. 1990;51(2):104-107.

6. Viola A, Nicoli F, Denis B, et al. High cerebral scyllo-inositol: a new marker of brain metabolism disturbances induced by chronic alcoholism. Magnetic Resonance Materials in Physics, Biology and Medicine. 2004;17(1):47-61.

7. Schweinsburg BC, Taylor MJ, Alhassoon OM, et al. Chemical Pathology in Brain White Matter of Recently Detoxified Alcoholics: A 1H Magnetic Resonance Spectroscopy Investigation of Alcohol-Associated Frontal Lobe Injury. Alcoholism: Clinical and Experimental Research. 2001;25(6):924-934. 
8. Schweinsburg BC, Taylor MJ, Videen JS, Alhassoon OM, Patterson TL, Grant I. Elevated myo-Inositol in Gray Matter of Recently Detoxified but Not Long-Term Abstinent Alcoholics: A Preliminary MR Spectroscopy Study. Alcoholism: Clinical and Experimental Research. 2000;24(5):699-705.

9. Meyerhoff DJ, Blumenfeld R, Truran D, et al. Effects of heavy drinking, binge drinking, and family history of alcoholism on regional brain metabolites. Alcoholism: Clinical and Experimental Research. 2004;28(4):650-661.

10. Meyerhoff DJ. Brain proton magnetic resonance spectroscopy of alcohol use disorders. In: Handbook of Clinical Neurology. Vol 125. Elsevier; 2014:313-337.

11. Ith $\mathrm{M}$, Bigler $\mathrm{P}$, Scheurer $\mathrm{E}$, et al. Observation and identification of metabolites emerging during postmortem decomposition of brain tissue by means of in situ $1 \mathrm{H}$-magnetic resonance spectroscopy. Magnetic Resonance in Medicine. 2002;48(5):915-920.

12. Kantarci K, Knopman DS, Dickson DW, et al. Alzheimer Disease: Postmortem Neuropathologic Correlates of Antemortem 1H MR Spectroscopy Metabolite Measurements. Radiology. 2008;248(1):210-220.

13. Heimer J, Gascho D, Chatzaraki V, et al. Postmortem ${ }^{1} \mathrm{H}-\mathrm{MRS}$-Detection of Ketone Bodies and Glucose in Diabetic Ketoacidosis. International Journal of Legal Medicine. 2018;132(2):593-598.

14. Thali MJ, Yen K, Schweitzer W, et al. Virtopsy, a new imaging horizon in forensic pathology: virtual autopsy by postmortem multislice computed tomography (MSCT) and magnetic resonance imaging (MRI)-a feasibility study. Journal of forensic sciences. 2003;48(2):386-403.

15. Bartha R. Effect of signal-to-noise ratio and spectral linewidth on metabolite quantification at 4 T. NMR in Biomedicine. 2007;20(5):512-521.

16. Kreis R. Quantitative localized $1 \mathrm{H}$ MR spectroscopy for clinical use. Progress in Nuclear Magnetic Resonance Spectroscopy. 1997;31(2):155-196.

17. Bottomley PA, Foster TH, Argersinger RE, Pfeifer LM. A review of normal tissue hydrogen NMR relaxation times and relaxation mechanisms from 1-100 MHz: dependence on tissue type, NMR frequency, temperature, species, excision, and age. Medical Physics. 1984;11(4):425-448.

18. Ruder TD, Hatch GM, Siegenthaler $L$, et al. The influence of body temperature on image contrast in post mortem MRI. European Journal of Radiology. 2012;81(6):1366-1370.

19. Suzuki S, Sakai O, Jara H. Combined volumetric T1, T2 and secular-T2 quantitative MRI of the brain: age-related global changes (preliminary results). Magnetic Resonance Imaging. 2006;24(7):877-887.

20. Fein G, Meyerhoff DJ, Weiner MW. Magnetic resonance spectroscopy of the brain in alcohol abuse. Alcohol Health and Research World. 1995;19:306-314.

21. Sammi MK, Pan JW, Telang FW, et al. Measurements of human brain ethanol T2 by spectroscopic imaging at 4 T. Magnetic Resonance in Medicine. 2000;44(1):35-40.

22. Mason GF, Bendszus M, Meyerhoff DJ, et al. Magnetic resonance spectroscopic studies of alcoholism: from heavy drinking to alcohol dependence and back again. Alcoholism: Clinical and Experimental Research. 2005;29(1):150-158.

23. Dreher W, Leibfritz D. New method for the simultaneous detection of metabolites and water in localized in vivo $1 \mathrm{H}$ nuclear magnetic resonance spectroscopy. Magnetic Resonance in Medicine. 2005;54(1):190-195.

24. MacMillan EL, Chong DG, Dreher W, Henning A, Boesch C, Kreis R. Magnetization exchange with water and $\mathrm{T} 1$ relaxation of the downfield resonances in human brain spectra at 3.0 $\mathrm{T}$. Magnetic Resonance in Medicine. 2011;65(5):1239-1246.

25. Hock A, MacMillan EL, Fuchs A, et al. Non-water-suppressed proton MR spectroscopy improves spectral quality in the human spinal cord. Magnetic Resonance in Medicine. 2013;69(5):1253-1260.

26. Zoelch N, Hock A, Henning A. Quantitative magnetic resonance spectroscopy at 3T based on the principle of reciprocity. NMR in Biomedicine. 2018;31(5):e3875. 
27. Ashburner J, Barnes G, Chen C, et al. SPM8 manual. Functional Imaging Laboratory, Institute of Neurology. 2012.

28. Versluis MJ, Kan HE, van Buchem MA, Webb AG. Improved signal to noise in proton spectroscopy of the human calf muscle at $7 \mathrm{~T}$ using localized B1 calibration. Magnetic Resonance in Medicine. 2010;63(1):207-211.

29. Edden RA, Schär M, Hillis AE, Barker PB. Optimized detection of lactate at high fields using inner volume saturation. Magnetic Resonance in Medicine. 2006;56(4):912-917.

30. Henning A, Schär M, Kollias SS, Boesiger P, Dydak U. Quantitative magnetic resonance spectroscopy in the entire human cervical spinal cord and beyond at 3T. Magnetic Resonance in Medicine. 2008;59(6):1250-1258.

31. Bottomley PA. Spatial Localization in NMR Spectroscopy in Vivo. Ann NY Acad Sci. 1987;508(1):333-348.

32. Yablonskiy DA, Neil JJ, Raichle ME, Ackerman JJ. Homonuclear J coupling effects in volume localized NMR spectroscopy: pitfalls and solutions. Magnetic Resonance in Medicine. 1998;39(2):169-178.

33. de Matos NM, Hock A, Wyss M, Ettlin DA, Brügger M. Neurochemical dynamics of acute orofacial pain in the human trigeminal brainstem nuclear complex. Neurolmage. 2017;162:162-172.

34. Bydder M, Hamilton G, Yokoo T, Sirlin CB. Optimal phased-array combination for spectroscopy. Magnetic Resonance Imaging. 2008;26(6):847-850.

35. Klose U. In vivo proton spectroscopy in presence of eddy currents. Magnetic Resonance in Medicine. 1990;14(1):26-30.

36. Provencher SW. Estimation of metabolite concentrations from localized in vivo proton NMR spectra. Magnetic Resonance in Medicine. 1993;30(6):672-679.

37. Simpson R, Devenyi GA, Jezzard P, Hennessy TJ, Near J. Advanced processing and simulation of MRS data using the FID appliance (FID-A) - an open source, MATLAB-based toolkit. Magnetic Resonance in Medicine. 2017;77(1):23-33.

38. Loudon M, Parise J. Organic Chemistry. Macmillan Learning; 2015.

39. Gasparovic C, Song T, Devier D, et al. Use of tissue water as a concentration reference for proton spectroscopic imaging. Magnetic Resonance in Medicine. 2006;55(6):1219-1226.

40. Chiu T-M, Mendelson JH, Sholar MB, et al. Brain alcohol detectability in human subjects with and without a paternal history of alcoholism. Journal of Studies on Alcohol. 2004;65(1):16-21.

41. Flory GS, O'Malley J, Grant KA, Park B, Kroenke CD. Quantification of ethanol methyl $1 \mathrm{H}$ magnetic resonance signal intensity following intravenous ethanol administration in primate brain. Methods. 2010;50(3):189-198.

42. Jost G, Harting I, Heiland S. Quantitative single-voxel spectroscopy: The reciprocity principle for receive-only head coils. Journal of Magnetic Resonance Imaging. 2005;21(1):66-71.

43. Natt O, Bezkorovaynyy V, Michaelis T, Frahm J. Use of phased array coils for a determination of absolute metabolite concentrations. Magnetic Resonance in Medicine. 2005;53(1):3-8.

44. Kreis R, Ernst T, Ross B. Absolute quantitation of water and metabolites in the human brain. II. Metabolite concentrations. Journal of Magnetic Resonance, Series B. 1993;102(1):9-19.

45. Gelbke H, Lesch P, Spiegelhalder B, Schmidt G. Postmortale Alkoholkonzentrationen II. Die Alkoholkonzentrationen im Blut und Liquor cerebrospinalis. Blutalkohol. 1978;15(1).

46. Grüner O. Die Bedeutung des Körperwassers für die Verteilung des Alkohols im Organismus. Deutsche Zeitschrift für die gesamte gerichtliche Medizin. 1957;46(1):53-65.

47. Lijnema T, Huizenga J, Jager J, Mackor A, Gips C. Gravimetric determination of the water concentration in whole blood, plasma and erythrocytes and correlations with hematological and clinicochemical parameters. Clinica Chimica Acta. 1993;214(2):129-138.

48. Ernst T, Kreis R, Ross B. Absolute quantitation of water and metabolites in the human brain. I. Compartments and water. Journal of Magnetic Resonance, Series B. 1993;102(1):1-8.

49. Condon B, Patterson J, Jenkins A, et al. MR relaxation times of cerebrospinal fluid. Journal of Computer Assisted Tomography. 1987;11(2):203-207. 
50. Tofts P. Quantitative MRI of the brain: measuring changes caused by disease. John Wiley $\backslash \&$ Sons; 2005.

51. Ogg RJ, Kingsley PB. Optimized precision of inversion-recovery T1 measurements for constrained scan time. Magnetic Resonance in Medicine. 2004;51(3):625-630.

52. Spielman DM, Glover GH, Macovski A, Pfefferbaum A. Magnetic resonance spectroscopic imaging of ethanol in the human brain: A feasibility study. Alcoholism: Clinical and Experimental Research. 1993;17(5):1072-1077.

53. Hebold G. Die Bewertung von Alkoholbefunden im Liquor cerebro-spinalis. Deutsche Zeitschrift für die gesamte gerichtliche Medizin. 1959;48(2):257-263. 


\section{Tables}

Table 1 Characteristic data of the 15 corpses included in this study

\begin{tabular}{ccccc}
\hline Corpse & Sex & Age & Cause of Death & Estimated PMI [h] \\
\hline $\mathbf{1}$ & F & 74 & Internal hemorrhage & $4-12$ \\
$\mathbf{2}$ & M & 21 & Drug intoxication & $9-24$ \\
$\mathbf{3}$ & M & 20 & Asphyxia & $0.5-6$ \\
$\mathbf{4}$ & M & 53 & Internal hemorrhage & $1-4$ \\
$\mathbf{5}$ & M & 70 & Cardiac arrest & $0.5-3$ \\
$\mathbf{6}$ & M & 66 & CO intoxication & $1-3$ \\
$\mathbf{7}$ & M & 20 & Asphyxia & $0-0.25$ \\
$\mathbf{8}$ & M & 65 & Drug intoxication & $168-336$ \\
$\mathbf{9}$ & M & 39 & Drug intoxication & $5-11$ \\
$\mathbf{1 0}$ & M & 53 & Cardiac arrest & $0.5-2$ \\
$\mathbf{1 1}$ & F & 53 & Drug intoxication & $5-7$ \\
$\mathbf{1 2}$ & M & 57 & Cardiac arrest & $1-1.5$ \\
$\mathbf{1 3}$ & F & 75 & Cardiac arrest & $4-12$ \\
$\mathbf{1 4}$ & M & 25 & Drug intoxication & $2-4$ \\
$\mathbf{1 5}$ & M & 66 & Polytrauma & $6-12$ \\
\hline
\end{tabular}




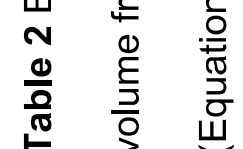




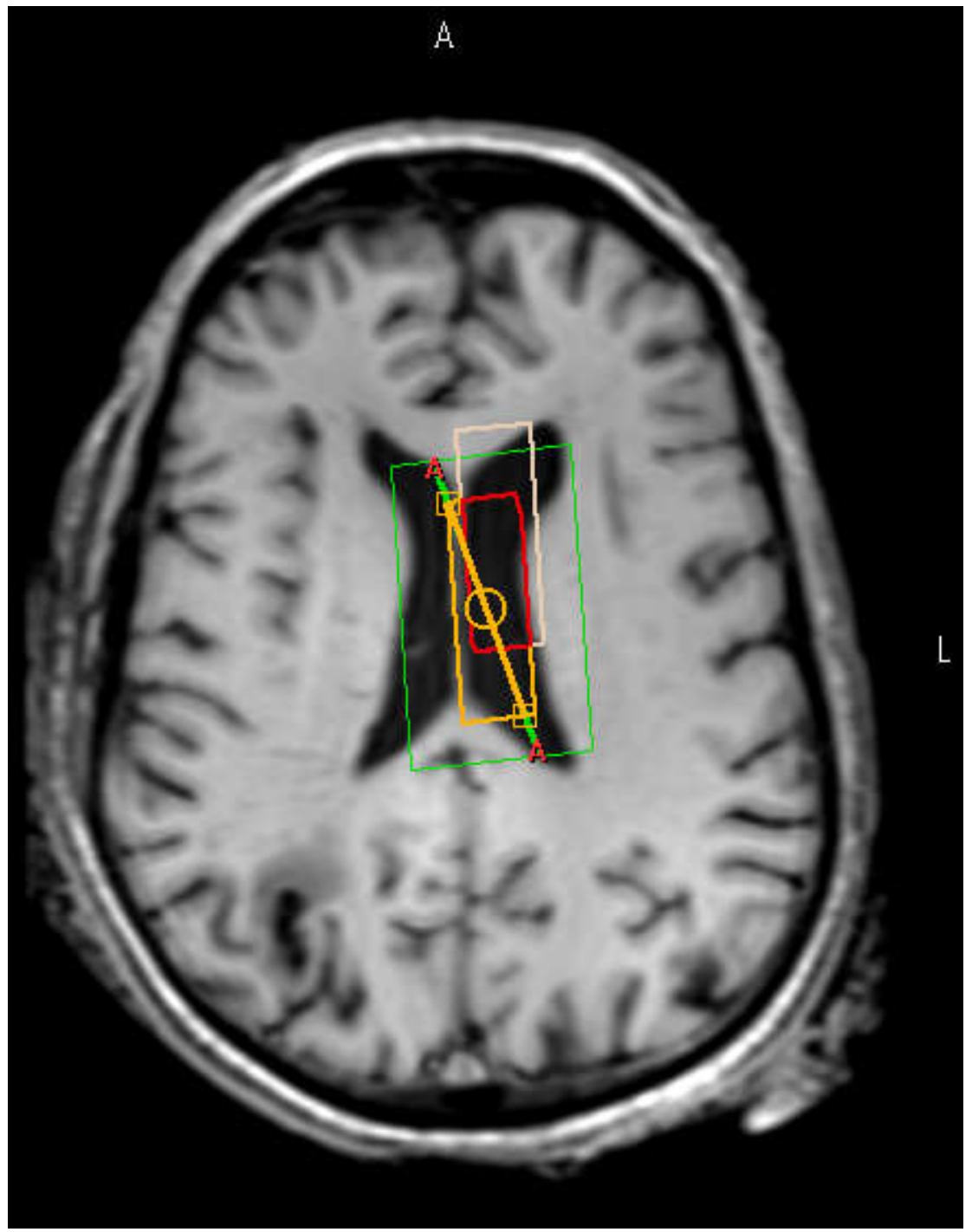

Figure 1: Exemplary voxel positioning in the left lateral ventricle. Orange and white boxes represent the acquisition volumes of lactate (1.32 ppm, orange) and water (white). The red box indicates the effective voxel size. The applied saturation bands for the inner-volume saturation are omitted. $\mathrm{B}_{0}$ shimming was performed within the volume represented by the green box.

$147 \times 189 \mathrm{~mm}(75 \times 75 \mathrm{DPI})$ 


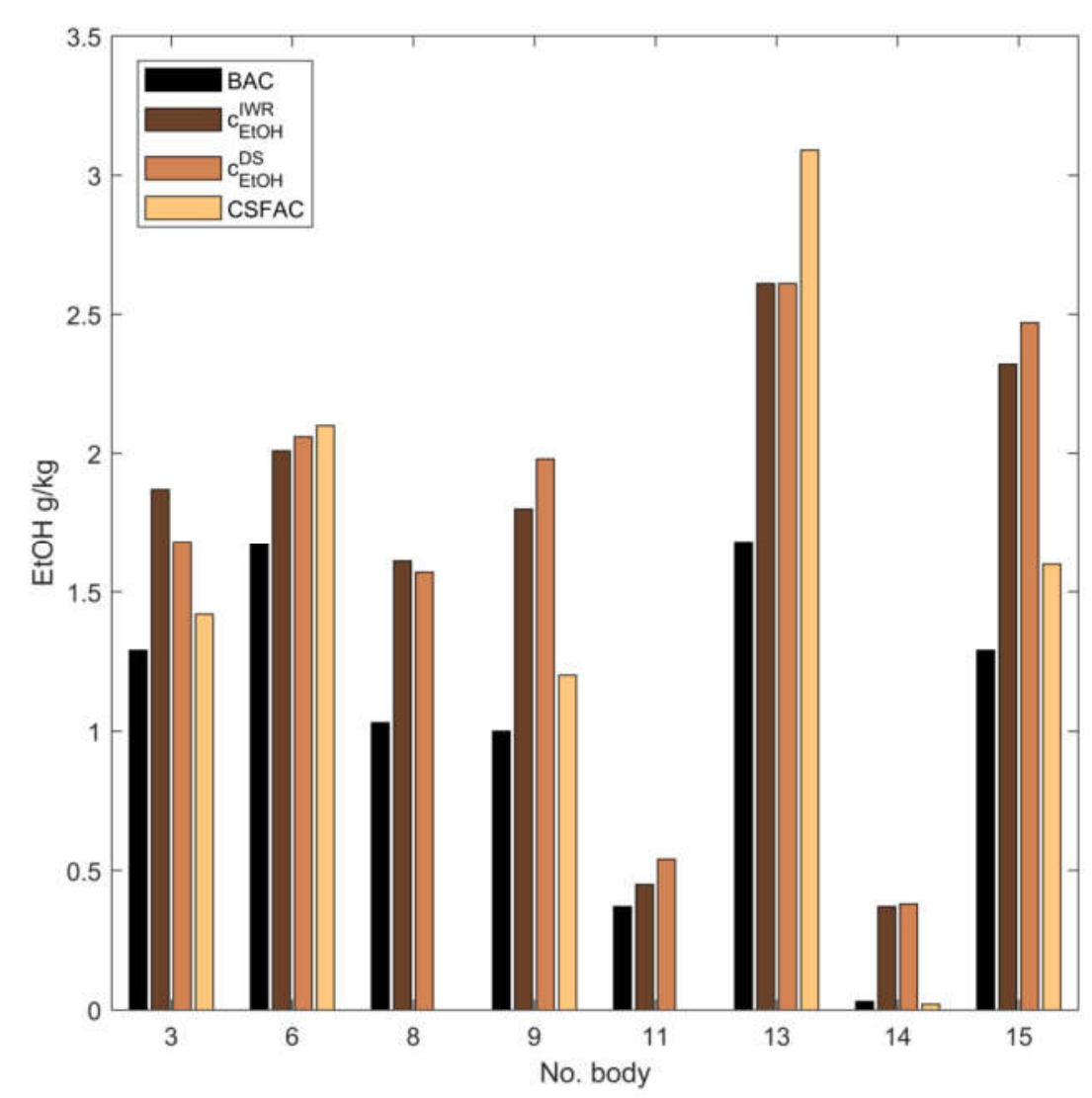

Figure 2: Graphical summary of the alcohol concentrations measured biochemically in whole blood (BAC) and CSF (CSFAC) and with MRS in CSF ( $\left.\mathrm{C}^{\mathrm{IWR}} \mathrm{EtOH}_{\text {and }} \mathrm{c}^{\mathrm{DS}}{ }_{\mathrm{EtOH}}\right)$.

$173 \times 166 \mathrm{~mm}(300 \times 300 \mathrm{DPI})$ 


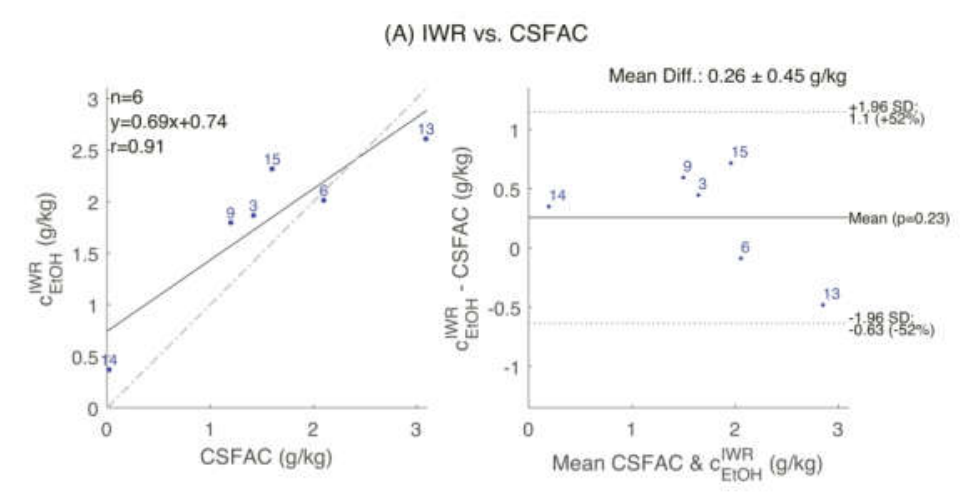

(B) IWR vs. BAC
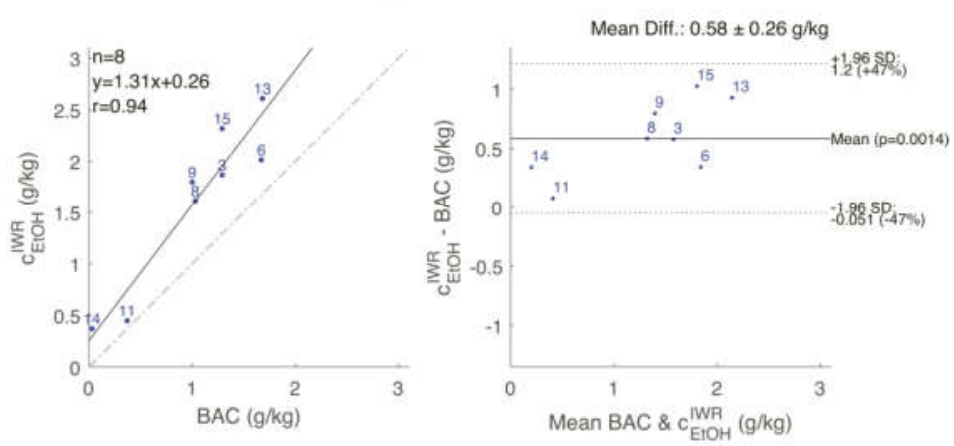

(C) DS vs. IWR
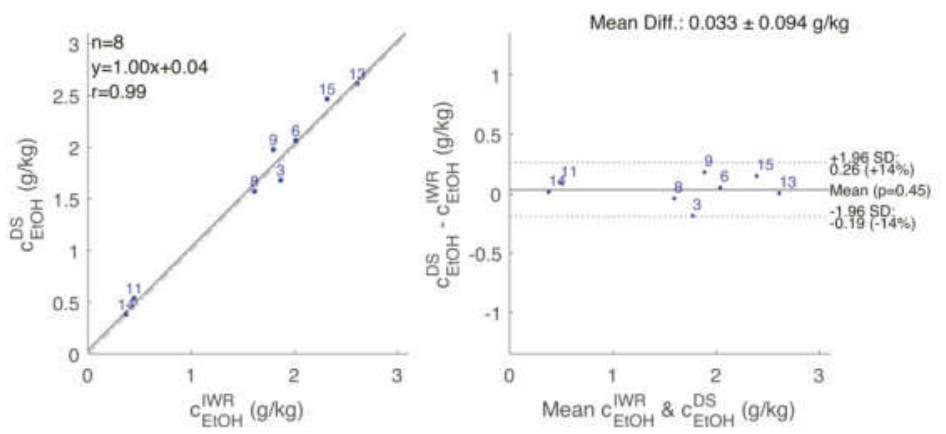

Figure 3: Scatter and Bland-Altman plots for different ethanol concentrations; $\mathrm{C}^{\mathrm{IWR}_{\text {EtOH }}}$ and CSFAC (A), $C^{I W R} E_{E t O H}$ and $B A C(B)$ and $c^{I W R} E_{E t O H}$ and $C^{D S}{ }_{E t O H}(C)$. In each scatter plot, the corresponding regression equation is shown together with the Pearson correlation coefficient. In the Bland-Altman plot, the mean difference and the upper and lower limits of agreement (LOA) are given. The LoA are additionally given as percentages of the average concentration value. Each data point is labeled with the corresponding subject number. 

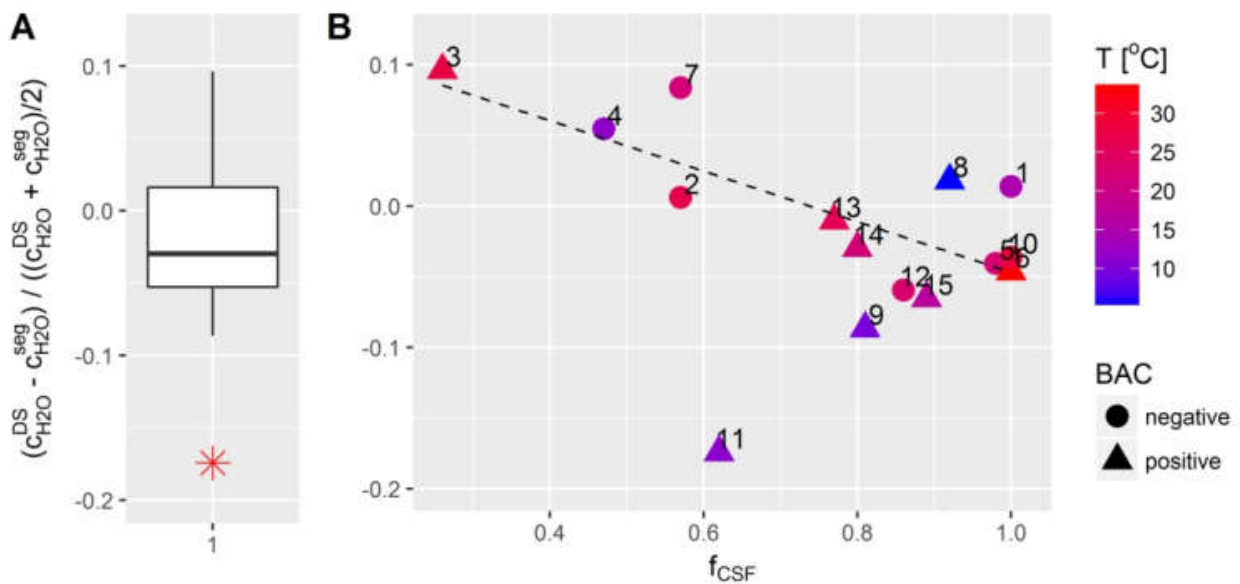

Figure 4: (A) Boxplot of the differences between the water concentrations $\mathrm{c}^{\mathrm{seg}} \mathrm{H}_{2 \mathrm{O}}$ and $\mathrm{C}^{\mathrm{DS}} \mathrm{H}_{2 \mathrm{O}}$ scaled with the mean of the two values. Outliers are marked with an asterisk. (B) The same values as in A plotted against the CSF fraction in the measured voxels. Subjects with a positive BAC are plotted with a triangle. The plotted line shows the regression line determined without the outlier No. 11.

$173 \times 82 \mathrm{~mm}(300 \times 300 \mathrm{DPI})$ 